\title{
EXPLORACIONES BOTÁNICAS A POBLACIONES DE CONSOLEA (CACTACEAE) EN CUBA: ESTADO DE CONSERVACIÓN Y PRINCIPALES AMENAZAS
}

\author{
Duniel Barrios \\ Grupo de Ecología y Conservación \\ Jardín Botánico Nacional \\ Universidad de La Habana, CUBA \\ duniel.barrios@gmail.com
}

\author{
Edgardo Díaz \\ Departamento de Biología \\ Facultad de Ciencias Agropecuarias, \\ Universidad Central "Marta Abreu" \\ de Las Villas Marta Abreu, CUBA \\ edalvarez@uclv.edu.cu
}

\author{
Lucas C. Majure \\ Florida Museum of Natural History \\ University of Florida \\ Gainesville, Florida 32611-7800, U.S.A. \\ Imajure@floridamuseum.ufl.edu
}

\section{RESUMEN}

Cuatro especies de Consolea han sido reportadas para el archipiélago cubano, todas consideradas como amenazadas de extinción. Durante los últimos tres años se realizaron varias expediciones con el fin de actualizar la información existente sobre el estado de conservación de sus poblaciones y sus principales amenazas. Se revisaron e identificaron los especímenes en los herbarios HAC, HAJB, HMC, MNHN y NY para elaborar un mapa de distribución por especie. Todas las especies de Consolea nativas de Cuba se mantienen bajo amenaza de extinción. Consolea moniliformis es la especie con mayores poblaciones mientras que C. macracantha tiene la mayor extensión de presencia; dos nuevas localidades fueron identificadas para esta especie que a su vez fue la única con evidencias de ataque por Cactoblastis cactorum. Entre las principales amenazas detectadas para Consolea en Cuba están las plantas exóticas invasoras, el pastoreo y las construcciones civiles. Proveemos una clave dicotómica para las especies de Cuba.

\section{ABSTRACT}

Four species of Consolea have been reported from the Cuban archipelago, each of which has been considered under threat of extinction. During the last three years various expeditions were carried out to try to update existing information about the conservation status of populations of the different species and their primary threats. Likewise, we revised and identified herbarium specimens from HAC, HAJB, HMC, MNHN, and NY to put together distribution maps for each species on Cuba. All native species of Consolea on Cuba are under threat of extinction/extirpation. Consolea moniliformis is the species with the largest populations on Cuba, while C. macracantha displays the widest range of any other species on the island. Two new localities of C. macracantha were found during this study, but likewise, showed the only signs to date of attack by the invasive moth, Cactoblastic cactorum. Among the principle threats detected for species of Consolea on Cuba are exotic, invasive plant species, livestock grazing and development. We provide a key to the species on Cuba.

Palabras Claves: Amenazas, Cactaceae, Cactoblastis cactorum, flor carpelada, flor estaminada

Consolea Lemaire es un género caribeño de cactus con hábito arbustivo o arbóreo, que representa un grupo monofilético (Areces-Mallea 1996; Majure et al. 2012) de nueve especies distribuidos desde los cayos de La Florida, Bahamas, y Antillas Mayores hasta la isla de Guadalupe en las Antillas Menores (Areces-Mallea 1996; Majure \& Clase 2018). Todas las especies de Consolea son poliploides (hexa u octaploides) (Negrón-Ortíz 2007), con un sistema sexual críptico dioico o subdioico (Strittmatter et al. 2006; Negrón-Ortíz 2007), que dificulta la producción de semillas y provoca en algunas especies que la propagación ocurra principalmente por vía asexual (Cariaga et al. 2005). En la evaluación global del estado de conservación de los cactus, Goettsch et al. (2015) solo consideraron 3 especies amenazadas de Consolea según los criterios de la UICN y ninguna era cubana.

En Cuba, cuatro especies de Consolea han sido reportadas (C. macracantha (Griseb.) A. Berger, C. millspaughii (Britton) A. Berger, C. moniliformis (L.) A. Berger y C. nashii (Britton) A. Berger), incluida una especie y dos subespecies endémicas (Areces-Mallea 1996). Las cuatro especies se distribuyen principalmente en las 
costas sur y norte del Oriente y algunos cayos del centro-norte de Cuba. A inicios de 2015 durante el II Taller de Conservación de Cactus Cubanos se recopiló información sobre el estado de las poblaciones de varias cactáceas cubanas con vistas a su recategorización (Barrios 2015). De la información compilada, la revisión de herbarios y literatura las cuatro especies de Consolea presentes en Cuba fueron reevaluadas como amenazadas (según los criterios de la IUCN v 3.1), tres como CR y una EN (Barrios \& Acuña 2015 a,b,c; Barrios et al. 2015). En los últimos tres años se han visitado algunas de las poblaciones de todas las especies de Consolea cubanas y se ha recopilado mayor información poblacional y de sus principales amenazas. En este sentido el principal objetivo de este artículo es actualizar la información existente sobre el estado de conservación de las poblaciones de todas las especies de Consolea nativas en Cuba.

Tres expediciones de campo fueron realizadas entre 2016-2018 (junio 2016, junio-julio 2017 y enerofebrero 2018) que abarcaron toda la Isla (Fig. 1). Durante las expediciones se anotaron observaciones como el número de individuos maduros de Consolea por localidad visitada, su sexo cuando era posible, y las amenazas observadas. En las especies C. macracantha y C. moniliformis debido a que es fácil distinguir a los individuos maduros (cuando no se presentan flores) de los estadios juveniles; se consideró juvenil a los individuos menores de $1.5 \mathrm{~m}$, preadulto entre 1.5-2.5 m, siempre que tuvieran las areolas de los cladodios superiores con numerosas espinas y como individuo maduro los individuos mayores de $2.0 \mathrm{~m}$ con los cladodios superiores mayormente sin espinas. En algunos casos donde la extensión de la población fue muy amplia, el número de individuos se estimó. Además de las observaciones de campo para cada especie se compiló e identificó todos los registros en herbarios cubanos y fuera de Cuba.

\section{Consolea macracantha (Griseb.) A. Berger}

1) Localidad.—El Salvial, Reserva Ecológica el Macío, Pilón Granma (W -7651'28.261"W, 1956'24.189"N) Formación vegetal._-Bosque seco degradado (potrero). La especie coexiste con Annona muricata L., Cereus peruvianus (L.) Mill., Croton sp., Harrisia fernowi Britton, Jatropha gossypiifolia L., Lantana sp., Malpighia sp., Melochia tomentosa L., Opuntia stricta (Haw.) Haw. var. dillenii (Ker Gawl.) L.D. Benson, Pilosocereus polygonus (Lam.) Byles \& G.D. Rowley, Stenocereus fimbriatus (Lam.) Lourteig, Senna atomaria (L.) H.S. Irwin \& Barneby, Tecoma stans (L.) Kunth, y las especies exóticas, invasoras Dichrostachys cinerea (L.) Wight \& Arn. y Kalanchoe daigremontiana Raym.-Hamet \& H. Perrier.

Información de la población.-Solo dos individuos preadultos entre 1.5-2.0 m de altura fueron observados en las cercanías de la Estación Ecológica (Majure, Barrios, Díaz 6998 - DES, HAJB; la única colecta de esta especie de la Prov. Granma). En el resto del área solo fueron observados alrededor de 10 individuos juveniles de menos de $50 \mathrm{~cm}$, probablemente propagados por vía asexual. Un tercer preadulto observado en 2015, había muerto en la visita al área del 2018. No se pudo determinar el sexo de individuos de esta población ya que no eran maduros.

Amenazas.—La principal amenaza en el área es la ganadería de bovinos y caprinos. Es muy probable que la escasez de individuos adultos se deba al corte por campesinos para evitar el daño al ganado. Otra amenaza es la graminización de los suelos y la invasión por otras especies exóticas como Dichrostachys cinerea y Kalanchoe daigremontiana. Uno de los dos individuos preadultos poseía cladodios con apariencia de ataque por Cactoblastis cactorum Berg., pero no se confirmó la presencia del pirálido en ninguno de los cladodios examinados.

\section{2) Localidad._Siboney, Santiago de Cuba (-75²43'11.716"W, 1957'34.87"N)}

Formación vegetal.-Matorral xeromorfo subcostero y bosque semideciduo microfilo. La especie coexiste con Adelia ricinella L., Agave underwoodii Trel., Commicarpus scandens (L.) Standl., Erythroxylum havanense Jacq., Exostema sp., Lantana buchii Urb., Malpighia sp., Pilosocereus polygonus, Randia aculeata L., Senna atomaria, Tecoma stans, Varronia bullata L. subsp. globosa (Jacq.) Greuter \& R. Rankin, así como las especies invasoras Abrus precatorius L. y Bothriochloa pertusa (L.) A. Camus.

Información de la población.-Siete individuos adultos fueron observados, tres de los cuales estaban 

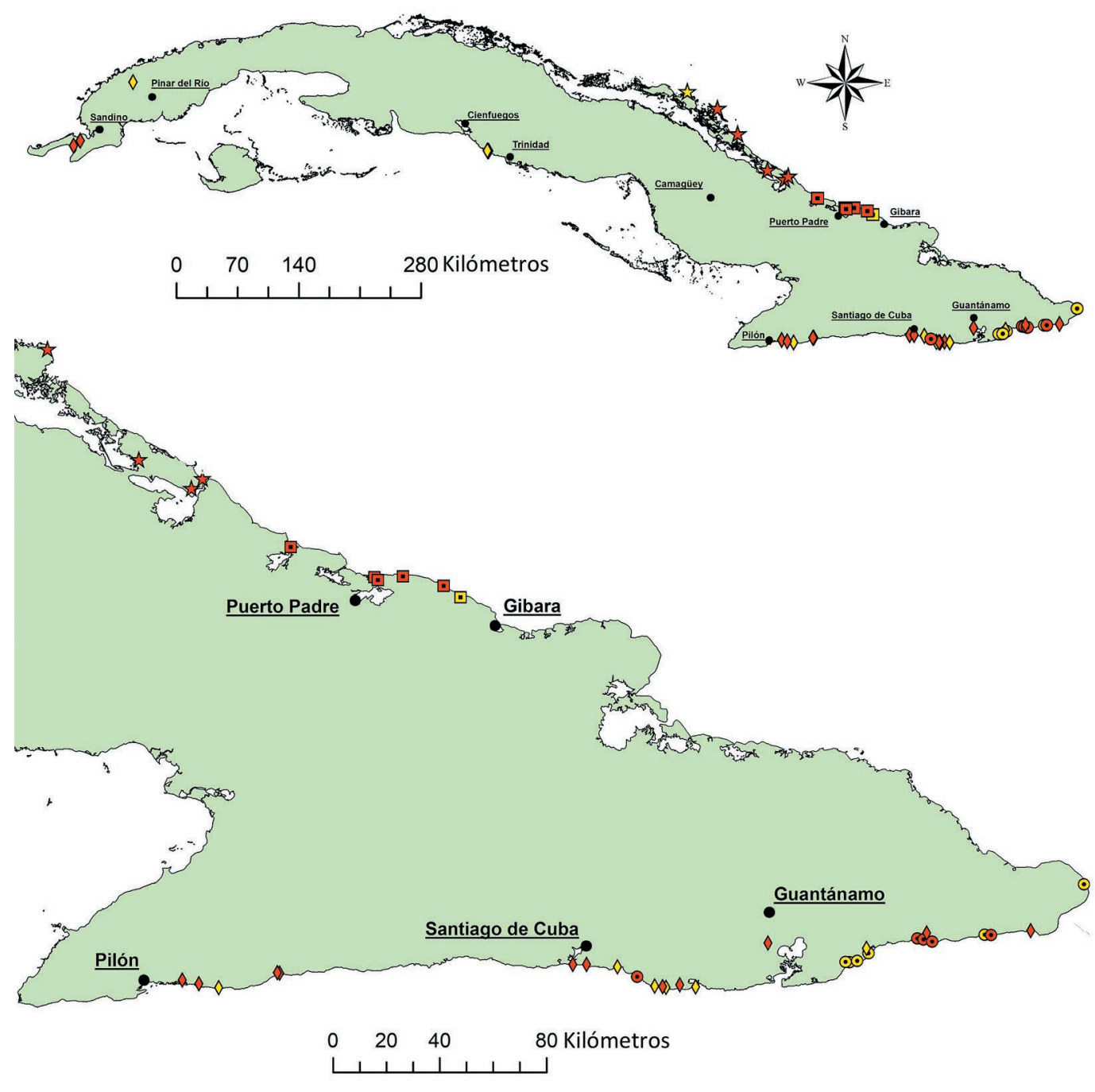

FIG. 1. Distribución de Consolea en Cuba. Consolea macracantha (diamantes); C. monilliformis subsp. guantanamana (círculos); C. nashii (cuadrados) y C. millspaughii (estrella). En amarillo las localidades visitadas y en rojo los especímenes de herbario. Los registros de dos localidades de C. macracantha entre Pilón y el Salvial, sin material de herbario fueron identificadas por fotos cedidas por J.M. Acuña y R.M. Verdecia.

florecidos y eran estaminados (Majure, Barrios, Díaz 7008 - DES, HAJB). Alrededor de 10 individuos preadultos fueron vistos y una decena de juveniles menores de $50 \mathrm{~cm}$ de altura.

Amenazas.-La principal amenaza en el área son los incendios, y en el 2017 una parte del área fue afectada.

3) Localidad._Playa el Papayo, Reserva Natural El Retiro, Santiago de Cuba (-75³5'41.587"W, 1953'40.506"N) Formación vegetal.-Matorral xeromorfo costero y bosque semideciduo micrófilo. Los individuos que crecen sobre arena se encuentran dentro de un bosque predominantemente de Phyllostylon rhamnoides (J. Poiss.) Taub., con otras especies como Amyris sp., Dendrocereus nudiflorus Britton \& Rose, Erythroxylum havanense y Acacia sp. Los individuos que crecen sobre roca se encuentran acompañados por Plumeria sp., Harrisia 
fernowii, Pseudocarpidium sp., Capparis flexuosa (L.) L., Pilosocereus brooksianus (Britton \& Rose) Byles \& Rowley y Randia aculeata.

Información de la población.-Es la población más grande de la especie observada hasta el momento (Barrios, Toledo, Palacio, Hernández, Díaz HFC89615 - HAJB). Alrededor de 50 individuos adultos fueron contabilizados e igual cantidad de juveniles. Los individuos se desarrollan tanto sobre roca caliza como en arena en $2 \mathrm{~km}$ de playa y costa rocosa. Un individuo estaminado de la especie fue localizado a $4 \mathrm{~km}$ al este de esta población cerca de playa Verraco (Majure, Barrios, Díaz 7005 - DES, HAJB).

Amenazas.-La principal amenaza para la especie en el área pudiera ser el impacto por huracanes. Si se tiene en cuenta que poblaciones de C. moniliformis que habitan ecosistemas similares y que han sido recientemente impactadas por huracanes muestran rasgos evidentes de afectación que pueden provocar hasta la muerte de los individuos adultos (ver: amenazas en la población de Cajobabo).

4) Localidad.—Baconao, Santiago de Cuba (-75³0'40.261"W, 1953'51.666"N)

Formación vegetal.-Vegetación ruderal contiguo al uveral compuesto por especies como Achyranthes aspera L., Guaiacum officinale L., Datura sp., Harrisia fernowi, Jatropha gossypiifolia, Opuntia militaris Britton \& Rose, O. stricta var. dillenii, Portulaca oleracea L., P. pilosa L., Prosopis juliflora (Sw.) DC., Sesuvium portulacastrum (L.) L., Stenocereus fimbriatus y las especies exóticas Dactyloctenium aegyptium (L.) Willd. y Dichrostachys cinerea.

Información de la población.-Esta población presenta una decena de individuos preadultos entre 1.5-2 m de altura, y alrededor de 40 juveniles menores de $50 \mathrm{~cm}$, aparentemente propagados por vía asexual (Majure, Barrios, Díaz 7003 - DES, HAJB). No se determinó el sexo de individuos de la población por su estado de desarrollo.

Amenazas.—Los individuos se encuentran en un área de matorral fragmentado con especies exóticas invasoras como Dichrostachys cinerea y varias gramíneas, también se han reforestado con Guaiacum officinale. Otras amenazas de importancia es el corte de los individuos probablemente para la limpieza de la plantación de G. officinale y el ataque por Cactoblastis cactorum (Fig. 2). Hasta el momento, solo existe un reporte de ataque de este pirálido en C. macracantha y C. moniliformis subsp. guantanamana en la Base Naval de Guantánamo (Areces-Mallea 2010) y anteriormente Stiling \& Moon (2001) habían confirmado la infestación en otra especie (C. corallicola en Little Torch Key). El actual reporte es el segundo en menos de un año de la infestación de C. cactorum en esta región en una especie de Cactaceae (subfamilia Opuntioideae) endémica. El primero fue sobre Opuntia militaris a solo $6 \mathrm{~km}$ al oeste de esta localidad (Barrios et al. 2017). Sin embargo, es notable que los individuos de O. militaris de Baconao aún no presentaban signos de infestación, solo algunos individuos de O. stricta sí se confirmaron infestados.

5) Localidad.—Baitiquirí, Guantánamo (-7452'48.428"W, 20¹'19.693"N)

Formación vegetal._Bosque semideciduo con Phyllostylon rhamnoides, Acacia sp., Ficus sp., Agave sp., Abutilon sp., Dichrostachys cinerea y Samanea saman (Jacq.) Merr. Sobre suelos pardo arcillosos profundos.

Información de la población.-Cinco individuos adultos y 4 preadultos fueron observados en un bosque cercano a un arroyo seco a $2.5 \mathrm{~km}$ de la costa. Alrededor de 10 juveniles al parecer por vía asexual fueron registrados. Los adultos florecidos eran estaminados (Majure, Barrios, Díaz 7016 - DES, HAJB). Esta subpoblación está en un hábitat más húmedo que donde se encuentran las subpoblaciones de C. moniliformis subsp. guantanamana y nunca se observó que convivan ambas especies, a pesar de estar muy cercanas.

Amenazas.-La principal amenaza de la especie es la competencia con especies exóticas invasoras como Dichrostachys cinerea, Acacia sp. y Bromelia pinguin L. Los individuos se encuentran en áreas de pastoreo y probablemente sean cortados por los campesinos para evitar el daño al ganado como también hacen con Cylindropuntia hystrix (Griseb.) Areces.

6) Localidad.-Yaguanabo, Cienfuegos $\left(-80^{\circ} 12^{\prime} 23.803^{\prime \prime} \mathrm{W}, 21^{\circ} 51^{\prime} 48.641^{\prime \prime} \mathrm{N}\right)$

Formación vegetal.-Matorral xeromorfo subcostero con Abutilon sp., Achyranthes aspera, Bursera simaruba (L.) Sarg., Capparisflexuosa, Erythroxylum havanense, Hibiscus phoeniceus Jacq., Melochia tomentosa, Pilosocereus 


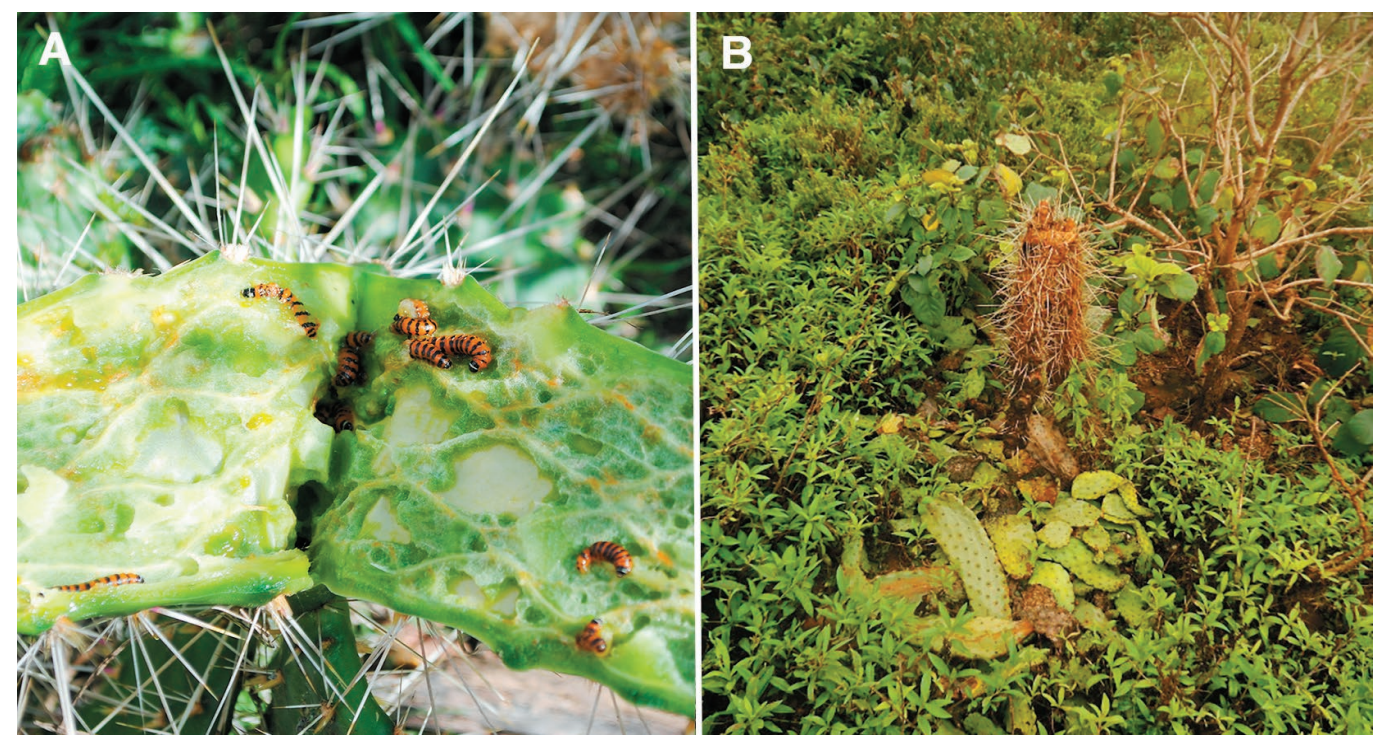

Fig. 2. A. Cladodio de C. macracantha infestado con larvas de Cactoblastis cactorum en Baconao, Santiago de Cuba. B. Individuo muerto de C. nashii en la carretera Gibara-Caletones, Holguín.

polygonus, Plumeria sp., Varronia bullata subsp. globosa, y las especies invasoras Dichrostachys cinerea y Leucaena leucocephala (Lam.) de Wit.

Información de la población.-En el área solo un individuo carpelado fue observado al lado de la carretera sobre una elevación de roca calcárea (Majure, Barrios, Díaz 7043 - DES, HAJB). Ganaderos de la zona comentan que existen otros individuos de la especie sobre rocas en los potreros, pero son muy escasos. En los herbarios HAJB (Álvarez y Gutiérrez HFC 69503) y MNHN (Areces 2404, 2423; Areces y Fleming 2949) existen colectas de especímenes del mismo lugar; solo el espécimen depositado en HAJB presenta una flor aparentemente hermafrodita. En esta región se requiere de mayor esfuerzo de muestreo para determinar el sistema sexual de otros individuos adultos.

Amenazas.-El área está a menos de cinco metros de la carretera y se encuentra completamente invadido por Leucaena leucocephala y Dichrostachys cinerea. El fuego es otra amenaza tanto real como potencial para esta población.

7) Localidad.—Chichones de los Indios, Viñales, Pinar del Río (-8352'8.387"W, 22³4'23.287"N)

Formación vegetal.—Bosque semideciduo con Cissus verticillata (L.) Nicolas \& C.E. Jarvis, Momordica charantia L., Oxandra lanceolata Baill., Plumeria sp., Selenicereus grandiflorus (L.) Britton \& Rose, Sideroxylum sp., Spondias mombin L., y Trichilia hirta L.

Información de la población.-Un solo preadulto de $1.70 \mathrm{~m}$ de altura fue observado sobre piedra caliza. Este individuo se encuentra a $4 \mathrm{~km}$ al noreste del poblado de Pons, en Minas de Matahambres y ningún otro individuo fue observado sembrado en las casas de los pobladores. Ninguna evidencia fue hallada que pudiera indicar que fuera un individuo introducido intencionalmente.

Amenazas.-La principal amenaza es la competencia durante el período lluvioso por trepadoras como Momordica charantia y Cissus verticillata. Durante el periodo lluvioso que se extiende de mayo a octubre, estas lianas limitan la luz que llega a los cladodios, lo cual puede provocar pudriciones en el individuo.

Notas.-Consolea macracantha es la especie más grande de Cuba formando árboles impresionantes de hasta $7 \mathrm{~m}$ de altura, con los troncos sumamente armados con espinas largas (Fig. 3A). La mayoría de 


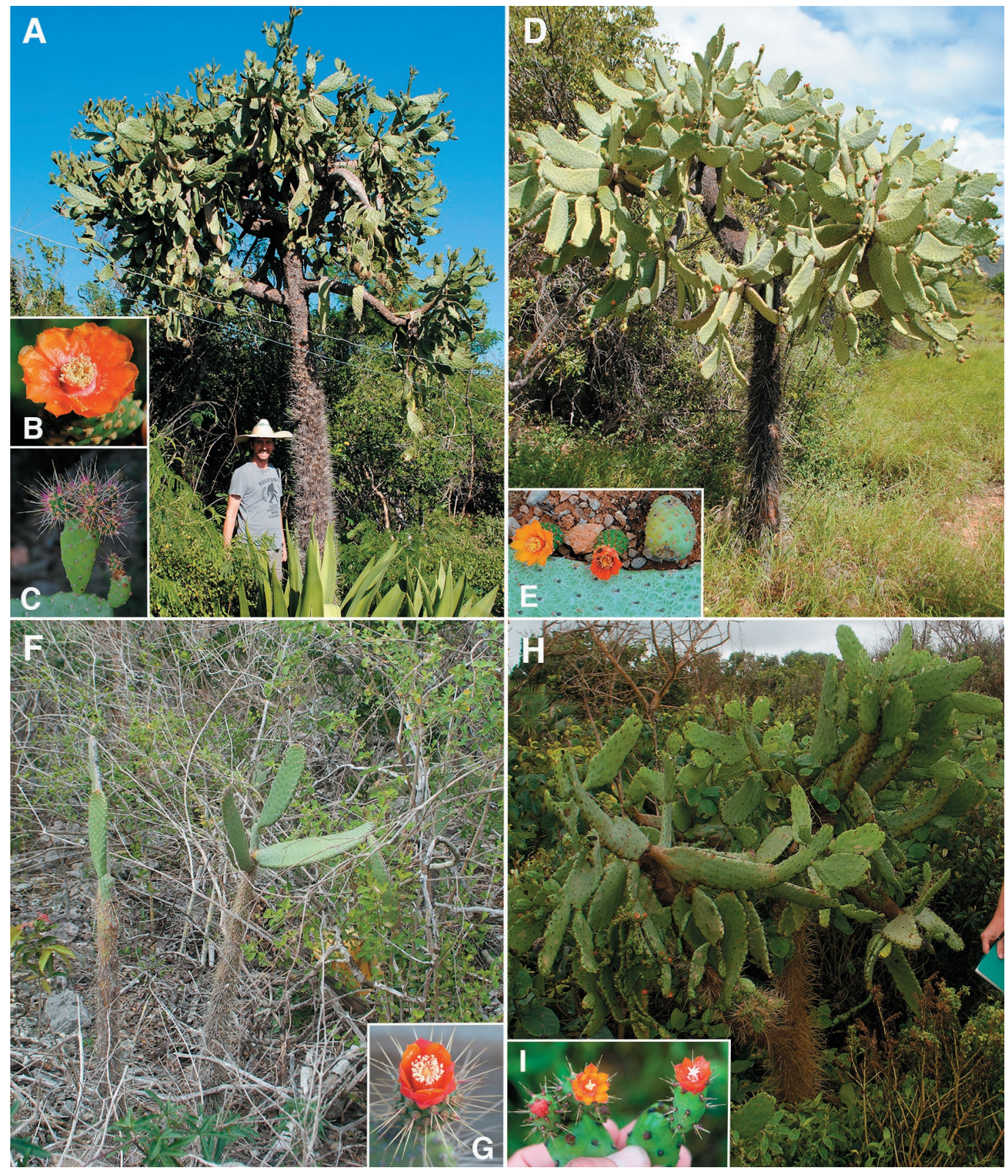

Fig. 3. Fotos de las cuatro especies de Consolea en Cuba. A. Árbol de Consolea macracantha en Verraco (Majure, Barrios, Díaz 7005); B. flor estaminada y pericarpelo sin espinas de las misma; C. fruto prolífero mostrando los cladodios nuevos, espinosos (Majure, Barrios, Díaz 7008); D. Árbol de C. moniliformis en Baitiquirí (Majure, Barrios, Díaz 7011); E. flores mostrando el cambio de color, fruto sin espinas y cladodio mostrando epidermis reticulada de la misma; F. arbusto de C. millspaughii (Majure, Barrios, Díaz 7034); G. flor de la misma mostrando el pericarpelo espinoso; H. arbusto (arbolito) de C. nashii ssp. gibarensis (Majure, Barrios, Díaz 7032); I. flores secundarias carpeladas de la misma mostrando el cambio de color de las flores, los frutos prolíferos y pericarpelo espinoso. 
poblaciones de C. macracantha parecen ser de plantas estaminadas (Fig. 3B), aunque encontramos una planta carpelada en Yaguanabo (Majure, Barrios, Díaz 7043) y R.M. Verdecia en enero de 2018 encontró otro adulto con frutos y semillas en el camino a Limones a $2.5 \mathrm{Km}$ al noreste de Punta Mota $\left(-77^{\circ} 07^{\prime} 28.28^{\prime \prime} \mathrm{W}\right.$, $19^{\circ} 54^{\prime 2} 25.266^{\prime \prime N}$ ), cerca de Pilón en la provincia Granma. Los frutos estériles de plantas estaminadas son prolíferos, formando cladodios sumamente espinosos que al caerse al suelo se desarrollan en una nueva planta (Fig. 3C).

Especímenes examinados (Fig. 1)._Prov. Cienfuegos: 1km al oeste de Yaguanabo, 14 abr 1991, Álvarez \& Gutiérrez HFC 69503 (HAJB) con flores hermafroditas. Lomas secas frente a Yaguanabo, sur del Escambray, 16 sep 1992, Areces 2404 (MNHN); Lomas secas frente a playa Yaguanabo, Prov. Cienfuegos, 6 ago 1993, Areces 2423 (MNHN); Yaguanabo, sur del Escambray, en loma rocosa cerca de la playa, 16 sep 1992, Areces \& Fleming 2949 (MNHN); En playa Yaguanabo a 200 metros de la comunidad de biplantas, en loma a un costado de la carretera Cienfuegos-Trinidad, 3 feb 2018, Majure, Barrios, Diaz 7043 (DES, HAJB). Prov. Granma: El Salvial, a 50 metros al sur de la Estación Biológica de la Reserva Ecológica El Macio, Pilón, N 19 53' 462" y W 77 04' 023", 24 ene 2018, Majure, Barrios, Díaz 6998 (DES, HAJB). Prov. Guantánamo: Yacabo, este de San Antonio del Sur, 2 feb 1976, Areces HFC 32021-24 (HAJB) con flor hermafrodita. Guantanamo Bay, Oriente, 17-30 Mar 1909, Britton 2057 (NY); Novaliches, Guantánamo, Oriente, 16 may 1918, Hioram 1808 (HAC, NY); Novaliches, Guantánamo, Oriente, nov 1919, Hioram s.n. (HAC); Montes cerca de Imías, 17 jul-4 aug 1924, León 12036 (HAC, NY); Novaliches, Guantánamo, Oriente 30 jun 1936, León 16730 (HAC); Lomas al norte de la Bahía de Baitiquirí, San Antonio del Sur, $20.01441^{\circ} \mathrm{N}$ y 74.89019O 88 m, 27 ene 2018, Majure, Barrios, Diaz 7016 (DES, HAJB); Guantanamo, Oriente, 23 feb 1911, Shafer 8909 (NY). Prov. Pinar del Río: Guanahacabibes, La Bajada, La Jaula, 3 nov 1973, Areces s.n. (HAJB); Guanahacabibes, La Bajada, 3 nov 1973, Areces, A. s.n. (HAJB); Camino a Chichones del Indio, al noreste del poblado de Pons, 28 jun 2016, Barrios \& Arias HFC 88792 (HAJB). Prov. Santiago de Cuba: Cuevas del turquino, 14 nov 1973, Areces HFC 32285 (HAJB); Cuevas del Turquino, al oeste de Ocujal, 16 nov 1973, Areces HFC 34127 (HAJB); Arroyo de Los Bueyes, al este de Playa Verraco, costa sur, 15 nov 1973, Areces HFC 34129 (HAJB); Terrazas costeras de Palenque, 2 km. al oeste de Playa Siboney, 14 nov 1976, Areces HFC 34135-36 (HAJB); Reserva Natural El Retiro, matorral xeromorfo costero, en playa El Papayo, 29 jul 2017, Barrios, Toledo, Palacio, Hernández \& Díaz HFC89615. Farallones del Morro de Santiago, Oriente, abr 1938, Clemente 18001 (HAC); Aguadores, Santiago, Oriente 11 abr 1940, León, Victorin, Clemente E Carabia 17685 (HAC); El Morro, Santiago, Oriente, oct 1938, León 18553 (HAC); Entre la Playa Casonal y La Laguna de Baconao a $200 \mathrm{~m}$ al oeste del restaurante Jaiba Azul, Baconao, 26 ene 2018, Majure, Barrios, Díaz 7003 (DES, HAJB); Tramo de la carretera entre Verraco-Sigua, aproximadamente $1 \mathrm{Km}$ al este de la playa de Verraco, 26 ene 2018, Majure, Barrios, Díaz 7005 (DES, HAJB); En la segunda terraza costera a ambos lados de las escaleras que suben hacia la estación biológica de la Reserva Ecológica Siboney-Juticí, al norte del poblado de Siboney, 26 ene 2018, Majure, Barrios, Díaz 7008 (DES, HAJB); Cuba (Santiago de Cuba) 1856-1860, Wright 2625 (HAC).

\section{Consolea moniliformis (L.) A. Berger subsp. guantanamana Areces}

\section{1) Localidad.-Tortuguilla-Baitiquirí (-74²52'29.203"W, $\left.20^{\circ} 0^{\prime} 20.278^{\prime \prime} \mathrm{N}\right)$}

Formación vegetal.—Matorral xeromorfo costero y subcostero, en ocasiones degradado. Entre las especies más comunes en esta zona se encuentran Agave sp., Colubrina elliptica (Sw.) Briz. \& W.L. Stern, Croton sp., Dendrocereus nudiflorus, Guaiacum officinale, Helicteres semitriloba Bert. ex DC., Lantana sp., Melocactus harlowii (Britton \& Rose) Vaupel, Melochia tomentosa, Oplonia tetrasticha (C. Wright) Stearn, Plumeria sp., Randia aculeata, Setaria sp., Selenicereus grandiflorus, Stenocereus fimbriatus, Varronia bullata subsp. globosa, Zanthoxylum sp. y la especie invasora Megathyrsus maximus (Jacq.) B.K. Simon \& S.W.L. Jacobs.

Información de la población.-La población de la especie se distribuye en una franja costera sobre diente de perro y suelos ferralíticos rojos de $12 \mathrm{~km}$ de largo. Los individuos adultos de la especie son abundantes y probablemente alcancen el millar. En enero de 2018 se observaron más de 10 individuos con flores hermafroditas al oeste de la Reserva Ecológica Baitiquirí que poseían frutos con semillas viables (ver Majure, Barrios, Díaz 7011 - DES, HAJB). Aunque la población de la especie en esta franja puede considerarse grande, en el futuro sería importante precisar hasta cuantos metros de la línea de costa se pueden distribuir los individuos de la especie, pues el mayor registro de distancia a la línea de costa es de $700 \mathrm{~m}$ tierra adentro, y por lo general se distribuyen en una estrecha franja que no sobrepasan los $500 \mathrm{~m}$.

Amenazas.-En toda el área hay pastoreo tanto bovino como caprino y son los individuos ubicados en los lugares más llanos los que poseen mayor peligro debido a que en ocasiones son cortados para no dañar el ganado. Gran parte de los matorrales originales de esta franja se han eliminado y aún persiste la tala en los relictos donde comienza el bosque siempreverde micrófilo. También la graminización por Megathrysus maximus (sin.=Panicum maximum) y la competencia con especies exóticas invasoras como la Leucaena leucocephala y Dichrostachys cinerea son amenazas que enfrenta la especie. Sin embargo, los individuos que crecen asociados 
a rocas calizas sobresalen junto a Stenocereus fimbriatus y debido a que estos suelos no tienen uso agrícola están en menor riesgo. El fuego puede ser otra amenaza recurrente en estos ecosistemas.

2) Localidad.-Playitas de Cajobabo $\left(-74^{\circ} 28^{\prime} 58.368^{\prime \prime} \mathrm{W}, 20^{\circ} 4^{\prime} 6.006^{\prime \prime} \mathrm{N}\right)$

Formación vegetal.-Matorral xeromorfo costero y bosque siempreverde micrófilo Agave sp., Calliandra colletioides, Cissus trifoliata, Evolvulus arbuscula Poir., Exostema caribaeum (Jacq.) Roem. \& Schult., Furcraea sp., Harrisia fernowi, Helicteres semitriloba, Jatropha gossypiifolia, Malpighia sp., Melochia tomentosa, Opuntia stricta var. dillenii, Phyllostylon rhamnoides, Pictetia sp., Plumeria sp., Setaria pradana (León ex Hitchc.) León, Stenocereus fimbriatus y las especies invasoras Bothriochloa pertusa y Leucaena leucocephala.

Información de la población.-En esta localidad un centenar de individuos adultos fueron observados e igual cantidad de individuos juveniles. Sin embargo, más de 20 individuos adultos fueron encontrados muertos al parecer por la pérdida de las ramas laterales debido al ciclón Matthew. Los que mantuvieron mayor número de ramas se encontraban más protegidos en la vegetación.

Amenazas.-En el área hay pastoreo, tala selectiva y un vertedero. La invasión por especies exóticas como Leucaena leucocephala aunque no está extendido es un problema. Los huracanes pueden provocar la muerte de los individuos adultos, si bien la especie deben estar adaptada a estos eventos meteorológicos, en poblaciones en declive y donde la vegetación ha sido alterada, el efecto de estos fenómenos naturales pudiera ser un serio problema para la persistencia de las poblaciones. Por ejemplo, recientes conteos de individuos del árbol Dendrocereus nudiflorus en la Punta de Maisí, reveló que el huracán Matthew fue responsable por la muerte de hasta 20\% de los individuos de 5-10 m de alto en áreas sin vegetación natural (Barrios, datos no publ.).

3) Localidad._Punta de Maisí (-748 $\left.{ }^{\prime} 50.859^{\prime \prime} \mathrm{W}, 20^{\circ} 14^{\prime} 15.36^{\prime \prime} \mathrm{N}\right)$

Formación vegetal.—matorral xeromorfo costero, muy bajo con Bothriochloa pertusa, Buxus sp., Caesalpinia sp., Calliandra colletioides, Cassytha filiformis, Cissus trifoliata, Clitoria sp., Croton sp., Erythroxylum sp., Eupatorium sp., Galactia sp. Heliotropium angiospermum, Helicteres semitriloba, Malpighia sp., Melochia tomentosa, Nama jamaicensis, Pilosocereus polygonus, Stenocereus fimbriatus, Pseudocarpidium sp, Paspalidium chapmanii, Stenocereus fimbriatus y Turnera sp.

Información de la población.-Solo tres individuos adultos fueron observados a unos $600 \mathrm{~m}$ al sureste del faro (Majure, Barrios, Díaz 7026 - DES, HAJB). Los individuos que no sobrepasaban $1.5 \mathrm{~m}$ de altura poseían muy pocas ramas laterales producto del ciclón Matthew. Según los trabajadores de la Reserva Ecológica MaisíCaleta, la especie (que conocen como alpargata) era antiguamente más abundante en la zona.

Amenazas.—La principal amenaza en esta área es el vertimiento de desechos sólidos, graminización y las construcciones civiles.

Notas.—Consolea moniliformis forma árboles, por lo general más pequeños que C. macracantha, y se reconoce fácilmente por su epidermis reticulada (Fig. 3D-E). Los individuos juveniles de C. moniliformis ssp. guantanamana tienen las espinas conspicuamente largas y presentan la epidermis muy poca reticulada dando la apariencia de juveniles de C. macracantha, por esta causa puede resultar difícil distinguir entre individuos juveniles de ambos taxones. En algunos casos la epidermis reticulada no se nota fuertemente en especímenes del herbario ya que durante el proceso de secado se pierde el patrón (e.g., Majure, Barrios, Díaz 7026). Por este hecho se requiere poner mucha atención a colectas de juveniles e individuos estériles de este taxón ya que las poblaciones son simpátricas con las de C. macracantha, y puede haber confusión en la identificación. Hemos visto que las flores de esta especie cambian de color, probablemente debido a la edad de la flor (Fig. 3E).

Especímenes examinados (Fig. 1)._Prov. Guantánamo: Imías, 14-15 sep 1952, Acuña 17864 (HAC); Meseta de Maisí, Baracoa, feb 1929 , Acuña 23926 (HAC); Costas frente a Playa Tortuguilla, al este de Guantánamo, 28 ene 1973, Areces HFC 23479-80 (HAJB); frutos con semillas, Costa entre playa Yateritas y Tortuguilla, 29 ene 1973, Areces HFC 23537, 23539-41 (HAJB); frutos con semillas, Tortuguilla, 12 dic 1973 , Areces HFC 24657 (HAJB); La Guardarraya (de Macambo), por la carretera, a 8 km al oeste de Imías, 19 ago 1975, Areces HFC 28267-71 (HAJB); con flores hermafroditas, 2 km al este de Tortuguilla, 19 ago 1975, Areces HFC 28311-21 (HAJB); Daiquirí, sur de Oriente, en la costa rocosa, 24 mar 1976, Areces \& Díaz HFC 31139 (HAJB); Terrazas al este de Cajobabo, 13 feb 1976, Areces HFC 31989-90 (HAJB); fruto con semillas, Cajobabo, en el camino entre Cajobabo y Arroyón, 13 feb 1976, HFC 31991 (HAJB); Tortuguilla, 5 feb 1976, Areces HFC 31994 (HAJB); San Antonio del Sur, costas, 29 ene 1973, Areces HFC 32239 (HAJB); San Antonio del Sur, costas, 29 ene 1973, Areces HFC 3224032242 (HAJB); Baitiquirí-El Naranjo, prov. Guantánamo San Antonio del Sur, N20 00.344 W74 52.497, matorral xeromorfo costero, 27-60 
msm, 20 jun 2016, Barrios, Arias \& Acuña HFC 88769 (HAJB); con flores hermafroditas, Lomas detrás de la bahía de Baitiquirí, 40 msm en matorral xeromorfo subcostero, 23-24 jun 2017, Barrios, Toledo \& Palacio HFC 89602 (HAJB); con flores hermafroditas, Playitas de Cajobabo, 17 jun 1972, del Risco 27562 y 27534 (HAC); Sobre caliza de la costa Caletica-Jauco, 17 jul-4 ago 1924, León 11934 (HAC, NY); Imías, jul 1924, León 12446 (HAC, NY); Imías, Woods. Yunas, Jan 1925, León \& Pérez 12446 (NY); Sobre rocas del estero de La Maya, Maisí, Or. jul 1938, León \& Seifriz 18511 (HAC); Cultivada en el Guatao Hab., de una planta traída de Maisí, oct 1938, León 18554 (HAC); Segunda terraza de Maisí Oriente, 19 ago 1939, León, Victorin E Carabia 19096 (HAC); Pedregales de Maisí, nov 1934, López 16187 (HAC); Reserva Biológica Baitiquirí en la primera terraza en frente del Bate Bate, N20 00.344 W74 52.497, 27 m, 27 ene 2018, Majure, Barrios, Díaz 7011 (DES, HAJB); Punta de Maisí, a 600 m al suroeste del faro, Maisí, N20 14.560 W74 08.601, 17 m, 28 ene 2018, Majure, Barrios, Díaz 7026 (DES, HAJB); Maisí, jun 1939, Matos 19019 (HAC).

\title{
Consolea millspaughii (Britton) A. Berger subsp. millspaughii
}

Localidad.—Cayo Paredón Grande (-78'42.843"W, 22²8'46.29"N)

Formación vegetal.—matorral xeromorfo costero con especies como Amyris sp., Bourreria succulenta Jacq., Bursera simaruba, Coccothrinax litoralis León, Conocarpus erectus L., Erythroxylum sp, Eugenia sp., Exostema caribaeum, Erithalis fruticosa L., Jacquinia sp., Lantana sp., Metopium toxiferum (L.) Krug. \& Urb., Mosiera sp., Myrsine sp., Passiflora sp., Pithecellobium sp., Pilosocereus royenii, Pseudocarpidium sp., Sideroxylon celastrinum (Kunth) T.D. Pennington y Zanthoxylum flavum Vahl.

Información de la población.-Alrededor de 15 individuos (probablemente adultos) fueron observados. Todos los individuos se encontraban con escasos cladodios producto del ciclón Irma, y solo uno se encontró con botones (Majure, Barrios, Díaz 7034 - DES, HAJB). La vegetación en general sufrió grandes afectaciones de su cobertura por el ciclón y la especie que es bastante frágil no fue la excepción. En el 2012, Acevedo reportó para esta área 374 individuos incluidos 58 adultos (Barrios \& Acuña 2015).

Amenazas.-Actualmente el área está degradada, debido a la construcción de un hotel, aunque quedan relictos de vegetación que de no construirse sobre ellas constituiría un excelente refugio para la especie.

Notas.-Consolea millspaughii subsp. millspaughii es el taxón más frágil y pequeña de Consolea en Cuba formando arbustos por lo general menores a $2 \mathrm{~m}$ (Fig. 3F), pero en vegetación densa pueden llegar hasta $2.5 \mathrm{~m}$ de alto y es una de las dos especies que tiene el pericarpelo espinoso (Fig. 3G).

\begin{abstract}
Especímenes examinados (Fig. 1).-Prov. Camagüey: Playa El Muerto en la costa norte de Cayo Romano, 23 feb 1981, Álvarez et al. HFC 43893 (HAJB); Cayo Sabinal, camino del fuerte San Hilario a Sabana Larga, 11 ene 1976, HFC s.n. (HAJB); Cayo Sabinal, 10 may 1976, Areces HFC 32048 (HAJB) con fruto. Cayo Sabinal, camino del Jato a la Playa Sabinal, 8 ene 1976, Areces HFC 32135 (HAJB); Cayo Paredón Grande, camino entre el faro y el hotel en construcción, al este de Cayo Coco, 1 Feb 2018, Majure, Barrios, Díaz 7034 (DES, HAJB); Al oeste de alto del Puerto, Cayo Cruz, Esmeralda, Camagüey, 21 ene 2016, Martinez HFC 89708 (HAJB); Cayo Cruz, Costa Norte de Camagüey, matorral xeromorfo costero y subcostero 24 feb 1990, Pérez \& Parrada 5309 \& 5286 (ACAM); Playa Bonita, Cayo Sabinal, Nuevitas, matorral xeromorfo costero y subcostero 24 mayo 1989, Pérez Chappy, Vandame 5912 (ACAM); Cayo Sabinal, Nuevitas, Camagüey, bosque siempreverde, 1989 , Pérez 7772 (ACAM); Cayo Paredón Grande, 1909, Shafer 2758 (NY).
\end{abstract}

\section{Consolea nashii (Britton) A. Berger subsp. gibarensis Areces}

\section{Localidad.—Camino Gibara-Caletones (-76¹5'2.292"W, $\left.21^{\circ} 12^{\prime} 18.499^{\prime \prime} \mathrm{N}\right)$}

Formación vegetal.—Matorral xeromorfo costero- subcostero y bosque siempreverde micrófilo. La especie coexiste con Caesalpinia vesicaria L., Capparis cynophallophora L., Cissus trifoliata L., Coccoloba uvifera L., Cordia sebestena L., Dysphania ambrosioides (L.) Mosyakin \& Clemants, Furcraea tuberosa (Mill.) W.T. Aiton, Opuntia stricta var. dillenii, y Thespesia populnea (L.) Correa.

Información de la población.-Nueve individuos adultos (incluidos 2 carpelados y 3 estaminados) fueron observados cercanos a la vía que abarca 22 km de extensión (Majure, Barrios, Díaz 7031, 7032 - DES, HAJB). Se observó un individuo muerto con todas las ramas laterales aun verdes en el suelo (Fig. 2), muy parecido a lo que ocurre cuando hay muerte por Cactoblastis cactorum; pero no se confirmó la presencia del pirálido en ningún cladodio. En general en el área los individuos se distribuyen a escasos metros del camino cuya vegetación se encuentra por tramos muy degradados, aunque según González et al. (2017), C. nashii puede encontrarse en el bosque siempreverde micrófilo más adentro en la vegetación.

Amenazas.-Las principales amenazas para la especie son la competencia con especies exóticas invasoras como Casuarina equisetifolia L. y Agave fourcroydes Lem., la ampliación de la vía, limpia de redes eléctricas, 
ampliación del parque eólico, y clareo de la vegetación para el establecimiento de hornos de carbón y siembra. Esta y otras amenazas en el área fueron registradas recientemente por González et al. (2017).

Notas.-Consolea nashii en Cuba forma arbustos grandes o arbolitos (Fig. 3H) y es la otra especie que tiene el pericarpelo espinoso (Fig. 3I). Observamos el mismo cambio de colores de las flores que vimos en $C$. moniliformis (Fig. 3I). Areces (1996) mencionó que la subespecie de Cuba, C. nashii ssp. gibarensis, casi nunca tiene espinas en el pericarpelo y cuando hay, no más de una por areola. Observamos que los frutos mayores de C. nashii no tenían espinas, pero que las flores y botones podían o no tener espinas y en ciertos casos las flores secundarias poseían areolas con más de una espina (Fig. 3I; ver Areces 1996). Este carácter parece ser muy variable en las poblaciones de C. nashii en Cuba ya que poblaciones de C. nashii en Las Bahamas casi siempre tienen espinas en todos los pericarpelos (Majure obsv. pers.).

Especímenes examinados (Fig. 1)._Prov. Holguín: Puerto Padre, Playa Herradura, 9 nov 1973 Areces \& Bisse HFC 24540 (HAJB); Costa rocosa entre Gibara y Playa Caletones, 3 jul 1990, Areces-Mallea 2256 (NY); Costa rocosa al oeste de Gibara, 30 sep 1992 Areces 2407 (MNHN); Gibara, Maniguas entre playa Caletones y El Mangle, 20 abr 1987, Arias et al. HFC 61617 (HAJB); con flores hermafroditas según notas de campo, Caletones-Gibara, Bosque semideciduo micrófilo, N21 12.356 y W76 14.95319 jun 2016, Barrios \& Arias HFC 88761 (HAJB); con flores carpeladas, Gibara, sobre roca diente-perro, en la costa, jul 1936, León 16611 (HAC); Bahía de Manatí, ene 1937, León 16826 (HAC); Caletones, Gibara, Or., 8 abr 1940, León, Victorin, Carabia 17667 (HAC); Mono Ciego, Bahía de Manatí, Oriente abr 1939, León E Alain 18973 (HAC); Mono Ciego, Manatí, Oriente abr 1939 León \& Alain 19011 (HAC); Junto al camino Gibara-Caletones, despues del parque eólico, frente al mar muy cerca de Caletones, 31 ene 2018, Majure, Barrios, Díaz 7031 (DES, HAJB); Ibid, 31 ene 2018, Majure, Barrios, Díaz 7032 (DES, HAJB); Playa Herradura, J. Menendez, Las Tunas, matorral xeromorfo costero, 23 feb 1988, Méndez \& Verdecia HLT3483 (HMC); La Boca, el Socucho, Puerto Padre, Las Tunas, matorral xeromorfo costero, 23 jun 1991 Verdecia \& Brull HLT5309 (HMC); El Faro, el Socucho, Puerto Padre, Las Tunas, matorral xeromorfo costero y subcostero, 7 nov 1993 Verdecia HLT6991 (HMC); Playa Las Bocas, Puerto Padre, Las Tunas, matorral xeromorfo costero y subcostero, 4 jun 1997, Verdecia HLT7208 (HMC); Los ejemplares de herbario reportados por Areces (1996) en el MNHN (Areces 3002, 3071, 4264, 4266) no fueron observados en la colección.

\section{Estado de conservación de las especies de Consolea Cubanas}

Después de tres años de expediciones a poblaciones de Consoleas cubanas proponemos que su estado de conservación se mantenga como en la compilación de González-Oliva et al. (2015). Durante estos tres años nuevas amenazas han surgido como es el ataque por Cactoblastis cactorum y la confirmación de que la especie es cortada para evitar el daño al ganado. Además, poseer una perspectiva más cercana del tamaño de sus poblaciones nos podría permitir en el futuro proponer para las especies en Peligro Crítico Consolea nashii subsp. gibarensis y Consolea millspaughii el criterio C2a(i) (IUCN 2019) pues posiblemente sus poblaciones en Cuba no superen los 250 individuos adultos debido a la pérdida de hábitat en las últimas décadas. Sin embargo, para verificar este supuesto se tendrían que prospectar antes todas las localidades donde las especies han sido reportadas/ recolectadas.

Por último, es válido hacer una alerta sobre las poblaciones de Consolea moniliformis subsp. guantanamana que actualmente parecen ser abundantes en la franja costera desde Tortuguilla a Punta de Maisí; aunque su distribución no es continua. Su regeneración no es muy abundante, generalmente se observan individuos adultos o escasos juveniles menores a $50 \mathrm{~cm}$, probablemente por propagación vegetativa y los estados intermedios entre estos extremos son bastante raros. El futuro desarrollo proyectado para esta franja del sur de Guantánamo unido a los planes de forestación-reforestación con especies exóticas y el pastoreo, pudieran provocar afectaciones severas en la especie y diezmar significativamente el número de efectivos poblacionales.

\section{CLAVE PARA LAS ESPECIES DE CONSOLEA DE CUBA}

1. Plantas mayormente arbustos o arbolitos de $2 \mathrm{~m}$ de alto, hasta $2.5 \mathrm{~m}$ en vegetación densa, pericarpelo espinoso o a veces sin espinas, epidermis de los cladodios no reticulada.

2. Arbustos por lo general de hasta $1.5 \mathrm{~m}$ de alto, poco ramificados, ancho de los individuos siempre menor a la altura, 9-13 areolas por hilera diagonal en los cladodios, podarios no elevados, lisos alrededor, epidermis verde-glauco, espinas del cladodio finas, plantas con un fuerte olor a Aloe

2. Arbustos o arbolitos por lo general de hasta $2 \mathrm{~m}$ de alto, muy ramificados, ancho de los individuos igual o mayor a la altura, 4-7 areolas por hilera diagonal en los cladodios, podarios elevados, demarcados con un semi-círculo en la epidermis, epidermis verde oscuro o clorótico, espinas de los cladodios robustas, plantas sin olor fuerte 
1. Plantas mayormente árboles $2.5-7 \mathrm{~m}$ de alto, pericarpelo usualmente desnudo o con pocas espinas, epidermis de los cladodios reticulada o no.

3. Epidermis reticulada, pericarpelo a veces con pocas espinas, Guantánamo, Cuba, La Española, Isla Mona, Puerto Rico

3. Epidermis lisa, pericarpelo sin espinas, Cuba

C. moniliformis

C. macracantha

\section{AGRADECIMIENTOS}

Agradecemos la colaboración existente entre el Jardín Botánico Nacional, el Desert Botanical Garden y el Jardín del Instituto de Biología de la Universidad Autónoma de México que hicieron posible gran parte de las expediciones. El presente estudio forma parte de los resultados del proyecto: "Taxonomía de la familia Cactaceae en Cuba: Una contribución a su conservación." Queremos agradecer a la Empresa Flora y Fauna, a Planta! y a la Sociedad Cubana de Botánica por su apoyo antes y durante las expediciones. La ayuda en el campo de E. Palacio, A. Legra, C. Legra, A. Hernández, Y. Revilla, M. Deloncelet, A. Morales, S. Toledo, G. Begué, W. Carmenate, J.L. Gómez, Antonio, J.M. Acuña, S. Arias, E. Martínez, R. Chaves, J.A. Beltrán y R.M. Verdecia fueron de inestimable valor para la realización de las expediciones, al igual que el apoyo de los trabajadores y directivos de las áreas protegidas visitadas. Las expediciones se realizaron mediante los permisos LH175 AN (09), LH AN (110) 2015, AN (63) 2017, AN (64) 2017, AN (65) 2017 y 76/2017. Agradecemos a dos revisores por los comentarios de una versión previa del manuscrito. El financiamiento de este proyecto fue realizado en parte por el Jardín Botánico Nacional, National Geographic Society y Desert Botanical Garden, Phoenix, Arizona, EE.UU.

\section{REFERENCES}

Areces-Mallea, A. 1996. New taxa of Consolea Lemaire (Cactaceae: Opuntioideae) from Cuba. Brittonia 48:224-236.

Areces-MalleA, A. 2010. Investigation of grazing impacts on native flora, identification of invasive flora abundance and distribution, and presence of rare, native flora at Naval Station Guantanamo bay, Cuba. Naval Facilities Engineering Command Southeast, Jacksonville, Florida, USA.

BARRIOS, D. 2015. El Il taller de conservación de Cactus Cubanos: 10 años después. Bissea 9:1.

BARRIOS, D. \& J.M. AcuÑA. 2015. Consolea macracantha. Bissea 9:185-186.

BARRIOS, D. \& J.M. AcuÑA. 2015. Consolea millspaughii subsp. millspaughii. Bissea 9:187.

BARRIOS, D. \& J.M. AcuÑA. 2015. Consolea moniliformis subsp. guantanamana. Bissea 9:188.

BarRIOS, D., J.M. AcuÑA, \& J.L. GÓMEZ. 2015. Consolea nashii subsp. gibarensis. Bissea 9:189.

BarRios, D., A. HeRnÁndez, Y. ReVILLA, \& M. DeRoncelé. 2017. Invasión de Cactoblactis cactorum (Lepidoptera: Pyralidae) sobre Opuntia (Cactaceae) en Cuba. Revista Jard. Bot. Nac. Univ. Habana 38:143-146.

Cariaga, K.A., C.E. LeWIs, J. Machinski, S.J. Wright, \& J. Francisco-Ortega. 2005. Patterns of genetic diversity in the critically endangered Florida Key endemic Consolea corallicola Small (Cactaceae): Evidence from inter-simple sequence repeat (ISSRs) DNA polymorphisms. Caribbean J. Sci. 41:225-233.

Goettsch, B., C. Hilton-Taylor, G. Cruz-Piñon, J.P. Duffy, A. Frances, H.M. Hernández, R. Inger, C. Pollock, J. Schi'Ér, M. Superina, N. Taylor, M. Tognell, A.M. Abba, S. Arias, H.J. Arreola-Nava, M.A. Baker, R.T. Bárcenas, D. Barrios, P. Braun, C.A. Butterworth, A. Búrouez, F. Caceres, M. Chazaro-Basañez, R. Corral-Diaz, M.Valle Perea, P.H. Demaio, W.A.D. Barros, R. Durán, L.F. Yancas, R.S. Felger, B. Fitz-Maurice, W.A. Fitz-Maurice, G. Gann, C. Gómez-Hinostrosa, L.R. Gonzales-Torres, M.P. Griffith, P.C. Guerrero, B. Hammel, K.D. Hell, J.G. Hernández-Oria, M. Hoffmann, M.I. Ishihara, R. Keisling, J. Larocca, J.L. León de la Luz, C.R. Loaiza S., M. Lowry, M.C. Machado, L.C. Majure, J.G. Martinez Ávalos, C. Martorell, J. Maschinski, E. Méndez, R.A. Mittermeler, J.M. Nassar, V. Negrón-Ortiz, L.J. Oakley, P. Ortega-Baes, A.B. Pin Ferreira, D.J. Pinkava, J.M. Porter, R. Puente-Martínez, M. Smith, J.M. Sotomayor M. del C., S.N. Stuart, J.L. Tapia Muñoz, T. Terrazas, M. Terry, M. Trevisson, T. Valverde, T.R. Van Devender, M.E. Vélz-Pérez, H.E. Walter, S.A. Wyatt, D. Zappl, J.A. Zavala-Hurtado, \& K.J. Gaston. 2015. High proportion of cactus species threatened with extinction. Nature PI. DOI: 10.1038/NPLANTS.2015.142.

González, P.A., S. I. Suárez, O. Leyva, J. L. Gómez, \& W. Carmenate. 2017. Flora y vegetación de la Reserva Ecológica Caletones, Gibara, Holguín, Cuba. Rev. Jardín Botánico Nacional 38:15-40.

González-Oliva, L., L.R.González-Torres, A. Palmarola, D. Barrios, \& E.Testé, eds. 2015. Categorización de taxones de la flora de Cuba 2015. Bissea 9:3-707.

IUCN. 2019. The IUCN Red List of Threatened Species. Version 2019-1. http://www.iucnredlist.org. Downloaded on 21 March 2019. 
Majure, L.C., R. Puente, M.P. Griffith, W.S. Judd, P.S. Soltis, \& D.S. Soltis. 2012. Phylogeny of Opuntia s.s. (Cactaceae): Clade delineation, geographic origins, and reticulate evolution. Amer. J. Bot. 99:847-864.

MAJuRE, L.C. \& T. CLASE. 2018. Uso del nombre Consolea microcarpa versus Consolea picardae, una especie endémica de La Española. J. Bot. Res. Inst. Texas 12:75-80.

Negrón-Ortiz, V. 2007. Chromosome numbers, nuclear DNA content, and polyploidy in Consolea (Cactaceae), an endemic cactus of the Caribbean Islands. Amer. J. Bot. 94:1360-1370.

StiLIng, P. \& D.C. Moon. 2001. Protecting rare Florida cacti from attack by the exotic cactus moth, Cactoblastis cactorum (Lepidoptera: Pyralidae). Florida Entomol. 84:507-509.

Strittmatter, L.I., R.J. Hickey, \& V. Negrón-Ortíz. 2006. Comparative microsporangium development in male-fertile and male-sterile flowers of Consolea (Cactaceae): When and how does pollen abortion occur. Grana 45:81-100. 